\title{
Long working hours and cardiovascular mortality: a census-based cohort study
}

\author{
Gianfranco Alicandro $^{1} \cdot$ Paola Bertuccio ${ }^{2} \cdot$ Gabriella Sebastiani $^{1} \cdot$ Carlo La Vecchia $^{3} \cdot$ Luisa Frova $^{1}$
}

Received: 20 November 2019/Revised: 5 March 2020 / Accepted: 19 March 2020

(C) Swiss School of Public Health (SSPH+) 2020

\begin{abstract}
Objectives Long working hours have been associated with cardiovascular disease (CVD) mortality. However, results are inconsistent and large cohort studies are needed to confirm these findings.

Methods We conducted a census-based cohort study including 11,903,540 Italian workers aged 20-64 years, registered in the 2011 census, with a 5-year follow-up (2012-2016). We estimated cause-specific hazard ratios (cHRs) through Cox regression models to quantify the association between long working hours and CVD mortality.

Results Over 5 years of follow-up, 17,206 individuals died from CVD (15,262 men and 1944 women). Men working 55 or more hours per week had a cHR of 0.95 (95\% confidence interval, CI 0.89-1.02) for all CVDs, while women showed a cHR of 1.19 (95\% CI 0.95-1.49). Professional women working more than $55 \mathrm{~h}$ per week had a cHR of 1.98 (95\% CI 0.87-4.52).

Conclusions This study does not support an association between long working hours and CVD mortality among active Italian men, while it suggests a possible excess risk among women, although based on limited number of events.
\end{abstract}

Keywords Cardiovascular diseases $\cdot$ Mortality $\cdot$ Long working hours $\cdot$ Overtime work $\cdot$ Workers $\cdot$ Occupation

\section{Introduction}

Previous studies evaluating the association between long working hours and non-fatal cardiovascular events showed conflicting results (Hannerz et al. 2018a, b; Kivimaki et al. 2015a, 2017; Virtanen et al. 2010), while only a few studies assessed cardiovascular disease (CVD) mortality (Holtermann et al. 2010; O'Reilly and Rosato 2013; Slopen et al. 2012). Validated and precise estimates of this association

Electronic supplementary material The online version of this article (https://doi.org/10.1007/s00038-020-01361-y) contains supplementary material, which is available to authorized users.

Gianfranco Alicandro

gianfranco.alicandro@istat.it

1 Directorate for Social Statistics and Population Census, National Institute of Statistics (ISTAT), Viale Liegi 13, 00198 Rome, Italy

2 Department of Biomedical and Clinical Sciences L.Sacco, Università degli Studi di Milano, Milan, Italy

3 Department of Clinical Sciences and Community Health, Università degli Studi di Milano, Milan, Italy are, however, challenging for several reasons, including publication bias, reverse causation and residual confounding. Publication bias plays a major role in summarizing the findings since authors are more likely to publish their results if they find significant associations between long working hours and CVD. Reverse causation can also affect the results since people who had experienced a cardiovascular event may reduce their working hours. Moreover, education is an important confounder since overtime working is variable across levels of education, while CVDs are more frequent among those with low education (Grosch et al. 2006). Finally, the effect of long working hours is likely different across groups of workers who are exposed to different psychosocial work-related factors, such as different levels of job control, job demand and balance between efforts and rewards.

Some studies linked work-related stress to several CVD risk factors, including high blood pressure, hyperglycaemia, dyslipidaemia, obesity and metabolic syndrome (Chandola et al. 2006, 2008; Kivimaki et al. 2015b; Nyberg et al. 2013). However, the evidence is still inconclusive, and there is no single mechanism involved, but rather many factors contribute to increase the risk (Kivimaki and 
Kawachi 2015). Work-related stress may also exacerbate pre-existing cardiovascular conditions with consequent excess risk of premature death (Steptoe and Kivimaki 2013).

In this study, we aimed to assess and quantify the association between long working hours and CVD mortality in all active individuals and in different groups of workers according to the type of occupation, using data from a uniquely large Italian cohort.

\section{Methods}

\section{Study design}

We carried out a historical cohort study based on the record linkage between the 2011 Italian census and the mortality archives for the period 2012-2016. In the 2011 census, two questionnaires were used: a short form, including a limited number of questions necessary for compliance with the EU regulations, and a long form, with a larger set of questions on socio-economic aspects, including the number of hours usually worked per week. The long form was sent to all the households in the municipalities with less than 20,000 residents and to a sample (one-third) of the households in municipalities with 20,000 residents or more and in all provincial capitals. Participation to the census was mandatory, and the questionnaires were mailed out to all the households included in municipal registers. Respondents had the option of returning back questionnaires online, by mail or by delivery to municipal collection offices. The coverage of the 2011 census was high with an under-coverage rate of $1.07 \%$ estimated by the post-enumeration survey.

The starting time of the observation was set on 1 January 2012, resulting in a maximum follow-up time of 5 years. Further details of the record-linkage procedure can be found elsewhere (Alicandro et al. 2017).

This study is part of an extensive project of the Italian National Institute of Statistics. The project is included in the National Statistical Program and was approved by the Italian Data Protection Authority.

\section{Study population}

We selected all active subjects aged 20-64 years at the start of follow-up with available data on occupation. In order to remove the individuals who may be unable to work full time due to disabling conditions, we excluded unemployed people and those not in the labour forces as well as subjects who worked less than $35 \mathrm{~h}$ a week.

\section{Study variables}

The primary outcome of the study was mortality from all CVDs, whereas mortality from ischaemic heart and cerebrovascular diseases were considered as secondary outcomes. The 10th Revision of the International Classification of Diseases (ICD) was used to identify the groups of causes of death: all CVD (ICD-10 codes: I00I99), ischaemic heart (ICD-10 codes: I20-I25) and cerebrovascular diseases (ICD-10 codes: I60-I69).

The exposure variable considered in this study was the number of hours usually worked per week. We used the following classes: $35-40 \mathrm{~h}, 41-48 \mathrm{~h}, 49-54 \mathrm{~h}$ and 55 or more hours.

Age at follow-up, marital status, number of dependent children, geographic area of residence, education and occupation were considered as covariates. Age at follow-up was grouped in the following categories: 20-39, 40-49, 50-54, 55-59, 60-64, 65-69 years. Marital status included the following groups: singles, married, separated or divorced and widowed. The number of dependent children was obtained by counting the number of individuals aged less than 18 years living in the same household. The number of dependent children was grouped in three categories including none, one and two or more children. Regions of residence were grouped in three major areas: North, Centre, South and major islands. The highest attained level of education was grouped in four categories: no education or primary school, middle school, high school and university.

We categorized the type of occupation in the following classes: (1) highly qualified employees, (2) intermediate qualified employees, (3) less qualified employees, (4) selfemployees, (5) professionals, (6) entrepreneurs and (7) others. We derived these categories from a census question regarding the type of job the individual had in the week that preceded the census date. The type of job had the following options: (1) employee; (2) professional; (3) selfemployee; (4) entrepreneur; (5) fixed-term contract worker; (6) casual worker; (7) member of a cooperative; and (8) family worker. We further split the category of employees taking into account the skills required to perform their job. To this purpose, we used another census question that reflects the major groups of the International Standard Classification of Occupation (ISCO-08) (ILO 2012) with the following options: (1) non-skilled manual work; (2) personnel working in manufacturing, machinery, assembly lines or drivers; (3) skilled manual work; (4) agriculture and farming; (5) sales and service work; (6) clerical work; (7) intermediate qualified technical, administrative, sport or artistic activities; (8) highly qualified activities including management, intellectual, scientific and artistic activities; 
(9) management of private or public companies; and (10) armed forces. Then, we classified the employees in the occupation category 8 or 9 as "highly qualified employees", those in the occupation category $3,4,5,6$ and 7 as "intermediate qualified employees" and those in the category 1 and 2 as "less qualified employees". Finally, we grouped fixed-term contract workers, casual workers, members of a cooperative and family workers in a whole category named "other occupations". The full scheme used to derive the occupation category is reported in Table S1 (online Supplementary material).

\section{Statistical analysis}

For each cause of death, we computed age-standardized mortality rates based on the world standard population (Doll et al. 1982).

We estimated cause-specific hazard ratios (cHRs) and the corresponding 95\% confidence intervals (CI) using Cox proportional hazard models censoring the competing event to the date of occurrence. Mortality from causes different from the cause of interest was considered as competing event.

We fitted two different models: a partially adjusted model including age categories and weekly worked hours as independent variables and a fully adjusted model including also marital status, number of dependent children, geographic area of residence, the highest attained educational level and occupation. The number of hours worked was included in the model as categorical variable, and the category " $35-40 \mathrm{~h}$ " was considered as reference.

For a more flexible modelling of the relationship between hours worked and CVD mortality, we used natural splines with knots fixed at equally spaced intervals. Different models were tested with knots located from 0 to 5 equally spaced intervals. The final model was selected on the basis of the lowest value of the Akaike information criterion (AIC).

For all CVD mortality, we carried out a stratified analysis by type of occupation and number of dependent children (none vs. at least one child).

As a sensitivity analysis, we also estimated the cHR for other causes to be sure that the potential effect of long work hours was specific to CVD mortality. In addition, to control for reverse causation, i.e. subjects with pre-existent cardiovascular conditions which may reduce their working hours, we excluded the events occurred over the first 3 years of follow-up.

\section{Results}

The cohort included 11,903,540 individuals $(8,091,716$ men and 3,811,824 women). Of these, $4.9 \%$ worked 55 or more hours per week $(5.7 \%$ among men and $3.1 \%$ among women).

Table 1 shows the distribution of the sociodemographic variables across categories of weekly worked hours. In both sexes, the two extreme categories of weekly worked hours were older as compared to the intermediate categories. Mean age (standard deviation) was 43.2 years (11.1), 41.9 years $(10.5), 42.1$ years $(10.4)$ and 44.3 years (10.0) among men working 35-40, 41-48, 49-54 and $\geq 55 \mathrm{~h}$ per week, respectively. Corresponding figures among women were: 44.1 (10.1), 41.3 (10.2), 40.8 (10.6), 42.6 (10.3) and 43.8 (10.3). Among long hours workers, more individuals were married. Among men, long hours workers were more likely to have at least one child, while the opposite was found among women. In men, the frequency of university graduates was not different between those working $35-40 \mathrm{~h}$ and long hours workers, while it was lower among women working $55 \mathrm{~h}$ or more per week. In both sexes, more than $40 \%$ of long hours workers were self-employed, while $18.6 \%$ among men and $13.7 \%$ among women were entrepreneurs.

Over 5 years of follow-up, 84,837 deaths $(68,034$ men and 16,803 in women) occurred. Of those, 17,206 were due to CVDs (15,262 in men and 1944 in women). Table 2 gives the number of deaths and the rates from all CVDs, ischaemic heart, cerebrovascular, other $\mathrm{CV}$ and non-CV causes across type of occupation and category of weekly worked hours.

Table 3 shows the cHRs estimated for categories of hours worked per week using $35-40 \mathrm{~h}$ as reference. Among men, mortality from all CVD was slightly reduced in those working 55 or more hours (cHR from the partially adjusted model: $0.91,95 \%$ CI $0.85-0.97$, cHR from the fully adjusted model: $0.95,95 \%$ CI $0.89-1.02$ ). Similar results were found when ischaemic heart and cerebrovascular diseases were analysed separately. Women working $55 \mathrm{~h}$ or more showed a slight excess mortality from all CVDs (cHR 1.16, 95\% CI 0.93-1.43) and ischaemic heart diseases (cHR: 1.28, 95\% CI 0.88-1.87), though not statistically significant. Mortality from cerebrovascular diseases was similar to women working $35-40 \mathrm{~h}$ per week (cHR: 0.96, 95\% CI 0.63-1.47). After adjusting for geographic area of residence, marital status, number of dependent children, education and occupation, the cHRs did not materially change for all CVD, whereas decreased to 1.18 (95\% CI 0.79-1.76) for ischaemic heart diseases. A slight decrease in non-CVD mortality was observed among 


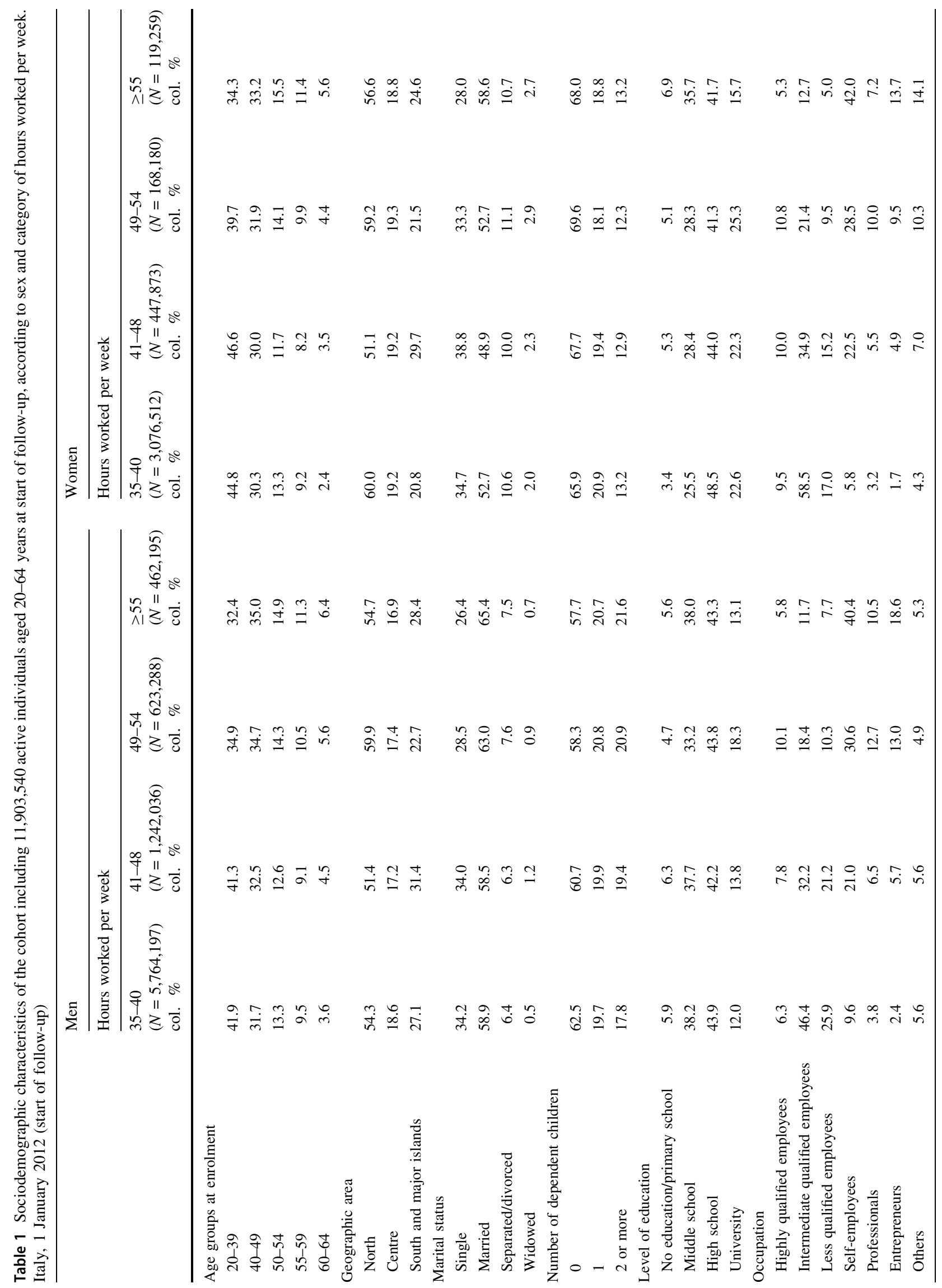


Table 2 Age-standardized mortality rates (number of deaths) from cardiovascular diseases and other non-cardiovascular causes per 100,000 person-years, according to sex and hours worked per week, in strata of occupation. Italy, 2012-2016

\begin{tabular}{|c|c|c|c|c|c|c|c|c|}
\hline & \multicolumn{4}{|l|}{ Men } & \multicolumn{4}{|l|}{ Women } \\
\hline & \multicolumn{4}{|c|}{ Hours worked per week } & \multicolumn{4}{|c|}{ Hours worked per week } \\
\hline & $35-40$ & $41-48$ & $49-54$ & $\geq 55$ & $35-40$ & $41-48$ & $49-54$ & $\geq 55$ \\
\hline \multicolumn{9}{|l|}{ All cardiovascular diseases } \\
\hline Highly qualified employees & $30.26(622)$ & $25.22(139)$ & $19.54(73)$ & $27.19(46)$ & $7.85(125)$ & $5.5(15)$ & $2.22(3)$ & $16.34(4)$ \\
\hline $\begin{array}{l}\text { Intermediate qualified } \\
\text { employees }\end{array}$ & 37.92 (4619) & $38.15(610)$ & $42.99(176)$ & $30.02(83)$ & $12.22(821)$ & $12.07(59)$ & $13.7(17)$ & $\begin{array}{r}29.38 \\
(12)\end{array}$ \\
\hline Less qualified employees & $47.06(3094)$ & $40.25(475)$ & $50.46(129)$ & $39.97(75)$ & $14.54(370)$ & $11.76(42)$ & $10.08(8)$ & $5.76(2)$ \\
\hline Self-employees & $40.16(1388)$ & $38.07(638)$ & $36.65(435)$ & $37.28(452)$ & $9.83(103)$ & $10.51(64)$ & $\begin{array}{r}10.58 \\
(36)\end{array}$ & $\begin{array}{r}11.66 \\
(37)\end{array}$ \\
\hline Professionals & $34.52(414)$ & $29.28(137)$ & $32.71(147)$ & $33.35(104)$ & $8.52(31)$ & $7.93(10)$ & $10.82(8)$ & $20.84(7)$ \\
\hline Entrepreneurs & $32.37(310)$ & $32.32(155)$ & $23.44(135)$ & $29.45(169)$ & $9.67(30)$ & $12.08(16)$ & $\begin{array}{r}14.22 \\
(11)\end{array}$ & $\begin{array}{r}13.28 \\
(13)\end{array}$ \\
\hline Others & $36.77(456)$ & $33.19(96)$ & $30.29(47)$ & $30.74(38)$ & $12.55(67)$ & $8.55(15)$ & $6.35(5)$ & $\begin{array}{r}11.41 \\
(13)\end{array}$ \\
\hline All occupations & $\begin{array}{l}39.03 \\
\quad(10,903)\end{array}$ & $\begin{array}{l}35.63 \\
\quad(2250)\end{array}$ & $\begin{array}{l}33.51 \\
\quad(1142)\end{array}$ & $34.15(967)$ & $11.7(1547)$ & $\begin{array}{l}10.54 \\
(221)\end{array}$ & $9.94(88)$ & $\begin{array}{r}13.53 \\
(88)\end{array}$ \\
\hline \multicolumn{9}{|l|}{ Ischaemic heart diseases } \\
\hline Highly qualified employees & $13.49(286)$ & $12.65(69)$ & $11.8(43)$ & $9.98(20)$ & $2.04(31)$ & $1.61(4)$ & $0.69(1)$ & $1.81(1)$ \\
\hline $\begin{array}{l}\text { Intermediate qualified } \\
\text { employees }\end{array}$ & $18.38(2245)$ & $18.38(277)$ & $22.57(93)$ & $14.91(43)$ & $3.28(226)$ & $2.74(13)$ & $7.32(8)$ & $1.91(1)$ \\
\hline Less qualified employees & $22.38(1488)$ & $20.17(228)$ & $27.27(65)$ & $16.5(29)$ & 4.27 (109) & $3.96(11)$ & $0.69(1)$ & $3.52(1)$ \\
\hline Self-employees & $19.75(695)$ & $18.39(309)$ & $16.96(218)$ & $16.82(208)$ & $3.9(42)$ & $2.47(15)$ & $3.08(10)$ & $5.17(14)$ \\
\hline Professionals & $17.43(205)$ & $14.46(70)$ & $15.32(66)$ & $18.89(58)$ & $1.91(8)$ & $4.66(5)$ & $2.73(2)$ & $12.3(2)$ \\
\hline Entrepreneurs & $14.99(146)$ & $14.81(71)$ & $11.77(66)$ & $16.38(98)$ & $4.09(12)$ & $5.97(7)$ & $5.47(4)$ & $7.06(5)$ \\
\hline Others & $17.44(222)$ & $12.88(39)$ & $11.45(17)$ & $17.88(21)$ & $1.93(15)$ & $2.6(5)$ & $2.16(1)$ & $4.6(5)$ \\
\hline All occupations & $18.79(5287)$ & $\begin{array}{l}17.13 \\
(1063)\end{array}$ & $16.23(568)$ & $16.67(477)$ & $3.28(443)$ & $3.04(60)$ & $3.39(27)$ & $5.11(29)$ \\
\hline \multicolumn{9}{|l|}{ Cerebrovascular diseases } \\
\hline Highly qualified employees & $3.18(66)$ & $3.82(24)$ & $2.89(11)$ & $3.48(6)$ & $2.57(43)$ & $0.89(3)$ & $0(0)$ & $1.81(1)$ \\
\hline $\begin{array}{l}\text { Intermediate qualified } \\
\text { employees }\end{array}$ & $5.33(606)$ & $6(98)$ & $6.48(22)$ & $3.33(12)$ & $3.29(243)$ & $3.27(22)$ & $4.69(5)$ & $11.45(5)$ \\
\hline Less qualified employees & $6.7(407)$ & $6.95(69)$ & $6.04(18)$ & $6.89(12)$ & $5.01(119)$ & $3.51(14)$ & $6.44(3)$ & $0(0)$ \\
\hline Self-employees & $5.38(182)$ & $5.34(87)$ & $5.21(59)$ & $5.46(70)$ & $3.11(33)$ & $3.2(20)$ & $3.98(15)$ & $1.22(6)$ \\
\hline Professionals & $5.65(70)$ & $3.19(15)$ & $4.11(18)$ & $2.1(8)$ & $1.66(9)$ & $0.72(1)$ & $1.87(1)$ & $4.42(3)$ \\
\hline Entrepreneurs & $4.35(44)$ & $4.2(20)$ & $3.18(18)$ & $3.23(16)$ & $2.48(9)$ & $3.27(4)$ & $1.09(1)$ & $1.96(3)$ \\
\hline Others & $5.95(64)$ & $9.12(21)$ & $3.03(6)$ & $2.32(3)$ & $4.3(21)$ & $4.84(8)$ & $3.63(3)$ & $4.3(4)$ \\
\hline All occupations & $5.42(1439)$ & $5.39(334)$ & $4.78(152)$ & $4.25(127)$ & 3.53 (477) & $3.22(72)$ & $3.2(28)$ & $2.93(22)$ \\
\hline \multicolumn{9}{|l|}{ Non-cardiovascular diseases } \\
\hline Highly qualified employees & $91.24(1944)$ & 77.9 (422) & $77.31(293)$ & $76.34(127)$ & $\begin{array}{c}80.72(1 \\
196)\end{array}$ & $61.6(136)$ & $68.3(64)$ & $\begin{array}{r}58.98 \\
(20)\end{array}$ \\
\hline $\begin{array}{l}\text { Intermediate qualified } \\
\text { employees }\end{array}$ & $\begin{array}{l}133.68 \\
(15,941)\end{array}$ & $\begin{array}{l}140.61 \\
(2202)\end{array}$ & $\begin{array}{r}138.57 \\
(629)\end{array}$ & $\begin{array}{r}151.25 \\
(336)\end{array}$ & $79.5(6504)$ & $\begin{array}{l}81.68 \\
(461)\end{array}$ & $\begin{array}{l}64.25 \\
(101)\end{array}$ & $\begin{array}{r}87.02 \\
(63)\end{array}$ \\
\hline Less qualified employees & $\begin{array}{l}162.65 \\
(10,762)\end{array}$ & $\begin{array}{l}169.18 \\
(1920)\end{array}$ & $\begin{array}{r}148.51 \\
(415)\end{array}$ & $\begin{array}{r}151.64 \\
(257)\end{array}$ & $\begin{array}{l}79.19(2 \\
294)\end{array}$ & $89.3(281)$ & $57.1(53)$ & $\begin{array}{r}89.23 \\
(32)\end{array}$ \\
\hline Self-employees & $142(4640)$ & $\begin{array}{l}129.94 \\
(2068)\end{array}$ & $\begin{array}{r}140.71 \\
(1548)\end{array}$ & $\begin{array}{r}136.07 \\
(1516)\end{array}$ & $83.43(891)$ & $\begin{array}{c}78.54 \\
(470)\end{array}$ & $\begin{array}{l}85.75 \\
\quad(268)\end{array}$ & $\begin{array}{l}85.29 \\
(273)\end{array}$ \\
\hline Professionals & $\begin{array}{l}106.56 \\
(1318)\end{array}$ & $98.47(466)$ & $\begin{array}{r}113.11 \\
(483)\end{array}$ & $96.51(310)$ & $77.87(305)$ & $89.8(97)$ & $\begin{array}{c}103.92 \\
(71)\end{array}$ & $\begin{array}{r}81.24 \\
(33)\end{array}$ \\
\hline Entrepreneurs & $\begin{array}{r}132.19 \\
(1129)\end{array}$ & $126.5(543)$ & $\begin{array}{r}131.96 \\
(599)\end{array}$ & $100.9(544)$ & $76.72(245)$ & 76.27 (99) & $\begin{array}{r}94.34 \\
(91)\end{array}$ & $\begin{array}{r}71.35 \\
(80)\end{array}$ \\
\hline
\end{tabular}


Table 2 (continued)

\begin{tabular}{|c|c|c|c|c|c|c|c|c|}
\hline & \multicolumn{4}{|l|}{ Men } & \multicolumn{4}{|l|}{ Women } \\
\hline & \multicolumn{4}{|c|}{ Hours worked per week } & \multicolumn{4}{|c|}{ Hours worked per week } \\
\hline & $35-40$ & $41-48$ & $49-54$ & $\geq 55$ & $35-40$ & $41-48$ & $49-54$ & $\geq 55$ \\
\hline Others & $132(1626)$ & $\begin{array}{r}146.86 \\
(403)\end{array}$ & $129.8(188)$ & $\begin{array}{r}108.78 \\
(143)\end{array}$ & $78.46(454)$ & $\begin{array}{c}72.96 \\
(115)\end{array}$ & $\begin{array}{r}63.15 \\
(74)\end{array}$ & $\begin{array}{r}96.88 \\
(88)\end{array}$ \\
\hline All occupations & $\begin{array}{c}136.52 \\
(37,360)\end{array}$ & $\begin{array}{c}134.05 \\
(8024)\end{array}$ & $\begin{array}{r}127.74 \\
(4155)\end{array}$ & $\begin{array}{r}122.87 \\
(3233)\end{array}$ & $\begin{array}{l}80.42 \\
\quad(11,889)\end{array}$ & $\begin{array}{c}79.66 \\
(1659)\end{array}$ & $\begin{array}{l}76.83 \\
(722)\end{array}$ & $\begin{array}{l}84.69 \\
(589)\end{array}$ \\
\hline
\end{tabular}

Table 3 Cause-specific hazard ratios (cHRs) and corresponding 95\% confidence intervals (CI) for mortality from cardiovascular and noncardiovascular diseases, according to sex and hours worked per week (reference category: 35-40 h). Italy, 2012-2016

\begin{tabular}{|c|c|c|c|c|c|}
\hline \multirow[t]{2}{*}{ Causes of death } & \multirow[t]{2}{*}{ Hours worked per week } & \multicolumn{2}{|l|}{ Men } & \multicolumn{2}{|l|}{ Women } \\
\hline & & $\begin{array}{l}\text { Model } 1 \\
\text { cHR }(95 \% \mathrm{CI})\end{array}$ & $\begin{array}{l}\text { Model } 2 \\
\text { cHR }(95 \% \mathrm{CI})\end{array}$ & $\begin{array}{l}\text { Model } 1 \\
\text { cHR }(95 \% \mathrm{CI})\end{array}$ & $\begin{array}{l}\text { Model } 2 \\
\text { cHR }(95 \% \mathrm{CI})\end{array}$ \\
\hline \multirow[t]{3}{*}{ All cardiovascular diseases } & $41-48$ & $0.93(0.89-0.98)$ & $0.93(0.89-0.98)$ & $0.98(0.85-1.13)$ & $0.96(0.83-1.11)$ \\
\hline & $49-54$ & $0.84(0.79-0.89)$ & $0.90(0.85-0.96)$ & $0.92(0.74-1.13)$ & $0.95(0.76-1.18)$ \\
\hline & $\geq 55$ & $0.91(0.85-0.97)$ & $0.95(0.89-1.02)$ & $1.16(0.93-1.43)$ & $1.19(0.95-1.49)$ \\
\hline \multirow[t]{3}{*}{ Ischaemic heart diseases } & $41-48$ & $0.91(0.85-0.97)$ & $0.91(0.85-0.97)$ & $0.93(0.71-1.22)$ & $0.86(0.65-1.14)$ \\
\hline & $49-54$ & $0.86(0.78-0.93)$ & $0.91(0.83-1.00)$ & $0.96(0.65-1.42)$ & $0.92(0.61-1.37)$ \\
\hline & $\geq 55$ & $0.91(0.83-1.00)$ & $0.95(0.86-1.05)$ & $1.28(0.88-1.87)$ & $1.18(0.79-1.76)$ \\
\hline \multirow[t]{3}{*}{ Cerebrovascular diseases } & $41-48$ & $1.05(0.93-1.18)$ & $1.05(0.93-1.18)$ & $1.04(0.81-1.34)$ & $1.02(0.79-1.32)$ \\
\hline & $49-54$ & $0.84(0.71-1.00)$ & $0.93(0.78-1.10)$ & $0.96(0.65-1.40)$ & $0.99(0.67-1.46)$ \\
\hline & $\geq 55$ & $0.90(0.75-1.08)$ & $0.95(0.79-1.15)$ & $0.96(0.63-1.47)$ & $0.98(0.62-1.53)$ \\
\hline \multirow{3}{*}{$\begin{array}{l}\text { Non-cardiovascular } \\
\text { diseases }\end{array}$} & $41-48$ & $0.98(0.95-1.00)$ & $0.98(0.96-1.01)$ & $0.97(0.92-1.02)$ & $0.95(0.90-1.00)$ \\
\hline & $49-54$ & $0.91(0.88-0.94)$ & $0.97(0.94-1.01)$ & $1.00(0.93-1.08)$ & $0.98(0.91-1.06)$ \\
\hline & $\geq 55$ & $0.91(0.88-0.94)$ & $0.95(0.92-0.99)$ & $1.05(0.97-1.14)$ & $1.02(0.94-1.11)$ \\
\hline
\end{tabular}

The cHRs were obtained from Cox regression models adjusted for age category (Model 1) and further adjusted for marital status, number of dependent children, geographic area, education and occupation (Model 2)

long working hours men, whereas no association was found among women.

When excluding the events occurred over the first three years, no association was found between working 55 and more hours per week and all CVD mortality among men (cHR: $0.98,95 \%$ CI $0.88-1.08$, based on 452 events among exposed men), whereas an excess mortality was observed among women (cHR: 1.26, 95\% CI 0.91-1.74, based on 44 events among exposed women).

The cHRs for all the variables included in the fully adjusted Cox regression model are shown in Table 4 . Mortality from CVD decreased with decreasing age and increasing level of education; it was lower among individuals living in North and Centre as compared to those living in the South of Italy, in those having at least one child and in those having highly qualified occupations, whereas mortality was higher among single, separated, divorced and widowed as compared to married individuals.

Figure 1 shows the relationship between hours worked per week and CVD mortality among men and women modelled as continuous and expressed as cHR (and corresponding 95\% CI) with $40 \mathrm{~h}$ per week used as reference. The relationship was not linear with some differences between sexes. In men, the risk of dying from CVDs was slightly higher for those working less than $40 \mathrm{~h}$, decreased for those working 40 or more and reached the same level of the reference at very high values. In women, the exposureresponse curve showed a decreasing risk until $55 \mathrm{~h}$ per week and a levelling off at higher values, although the small number of events yielded wide confidence intervals.

Figure 2 gives the cHRs for all CVD mortality among men and women working 55 or more hours per week compared to those working 35-40 h by type of occupation, obtained from the fully adjusted Cox regression model. The analysis stratified by type of occupation showed null associations among men. In women, all occupations showed an excess mortality among long hours workers, with the only exception of less qualified employees, although the precision of the estimates was low due to the small number of events. Professional women showed an 
Table 4 Cause-specific hazard ratios (95\% confidence intervals) for cardiovascular mortality by sex, estimated from multivariable Cox regression model. Italy, 2012-2016

\begin{tabular}{|c|c|c|}
\hline & Men & Women \\
\hline \multicolumn{3}{|l|}{ Age category } \\
\hline $20-39$ & $0.024(0.022-0.026)$ & $0.03(0.02-0.04)$ \\
\hline $40-49$ & $0.11(0.10-0.12)$ & $0.13(0.10-0.16)$ \\
\hline $50-54$ & $0.25(0.23-0.26)$ & $0.26(0.21-0.31)$ \\
\hline $55-59$ & $0.40(0.38-0.43)$ & $0.36(0.29-0.44)$ \\
\hline $60-64$ & $0.65(0.61-0.69)$ & $0.62(0.51-0.75)$ \\
\hline $65-69$ & 1 (Reference) & 1 (Reference) \\
\hline \multicolumn{3}{|l|}{ Education } \\
\hline No education or primary school & 1 (Reference) & 1 (Reference) \\
\hline Middle school & $0.88(0.83-0.94)$ & $0.77(0.66-0.90)$ \\
\hline High school & $0.75(0.70-0.80)$ & $0.69(0.58-0.81)$ \\
\hline University & $0.59(0.54-0.65)$ & $0.49(0.39-0.61)$ \\
\hline \multicolumn{3}{|l|}{ Geographic area } \\
\hline South and major islands & 1 (Reference) & 1 (Reference) \\
\hline Centre & $0.83(0.79-0.87)$ & $0.89(0.78-1.01)$ \\
\hline North & $0.78(0.75-0.81)$ & $0.78(0.70-0.87)$ \\
\hline \multicolumn{3}{|l|}{ Marital status } \\
\hline Married & 1 (Reference) & 1 (Reference) \\
\hline Singles & $1.63(1.56-1.70)$ & $1.42(1.26-1.61)$ \\
\hline Separated/divorced & $1.55(1.48-1.64)$ & $1.36(1.19-1.54)$ \\
\hline Widowed & $1.79(1.59-2.00)$ & $1.36(1.12-1.65)$ \\
\hline \multicolumn{3}{|l|}{ Number of dependent children } \\
\hline 0 & 1 (Reference) & 1 (Reference) \\
\hline 1 & $0.89(0.85-0.93)$ & $0.70(0.60-0.82)$ \\
\hline 2 or more & $0.83(0.78-0.88)$ & $0.83(0.68-1.02)$ \\
\hline \multicolumn{3}{|l|}{ Occupation } \\
\hline Less qualified employees & 1 (Reference) & 1 (Reference) \\
\hline Highly qualified employees & $0.84(0.77-0.92)$ & $0.86(0.69-1.07)$ \\
\hline Intermediate qualified employees & $0.89(0.85-0.92)$ & $0.92(0.81-1.05)$ \\
\hline Self-employees & $0.93(0.88-0.98)$ & $0.84(0.71-0.99)$ \\
\hline Professionals & $0.97(0.89-1.06)$ & $0.98(0.73-1.33)$ \\
\hline Entrepreneurs & $0.80(0.74-0.87)$ & $0.92(0.71-1.19)$ \\
\hline Others & $0.87(0.80-0.95)$ & $0.86(0.69-1.08)$ \\
\hline \multicolumn{3}{|l|}{ Hours worked per week } \\
\hline $35-40$ & 1 (Reference) & 1 (Reference) \\
\hline $41-48$ & $0.93(0.89-0.98)$ & $0.96(0.83-1.11)$ \\
\hline $49-54$ & $0.90(0.85-0.96)$ & $0.95(0.76-1.18)$ \\
\hline$\geq 55$ & $0.95(0.89-1.02)$ & $1.19(0.95-1.49)$ \\
\hline
\end{tabular}

increased mortality from all CVD $(1.98,95 \%$ CI $0.87-4.52$ in the fully adjusted model).

The interaction term between hours worked per week and having at least one dependent child was not statistically significant in both men $(P=.751)$ and women $(P=.013)$. The cHRs for men working more than $55 \mathrm{~h}$ per week were 0.88 (95\% CI 0.76-1.01) among men with at least one dependent child and 0.97 (95\% CI 0.90-1.06) in those without children. Corresponding figures for women were 0.78 (95\% CI $0.39-1.56)$ and 1.27 (95\% CI 1.00-1.62) for women with at least one dependent child and for those without children, respectively.

\section{Discussion}

In this study, long working hours were not associated with increased CVD mortality among men, while the results suggest a possible excess risk among women, although based on a limited number of events. 


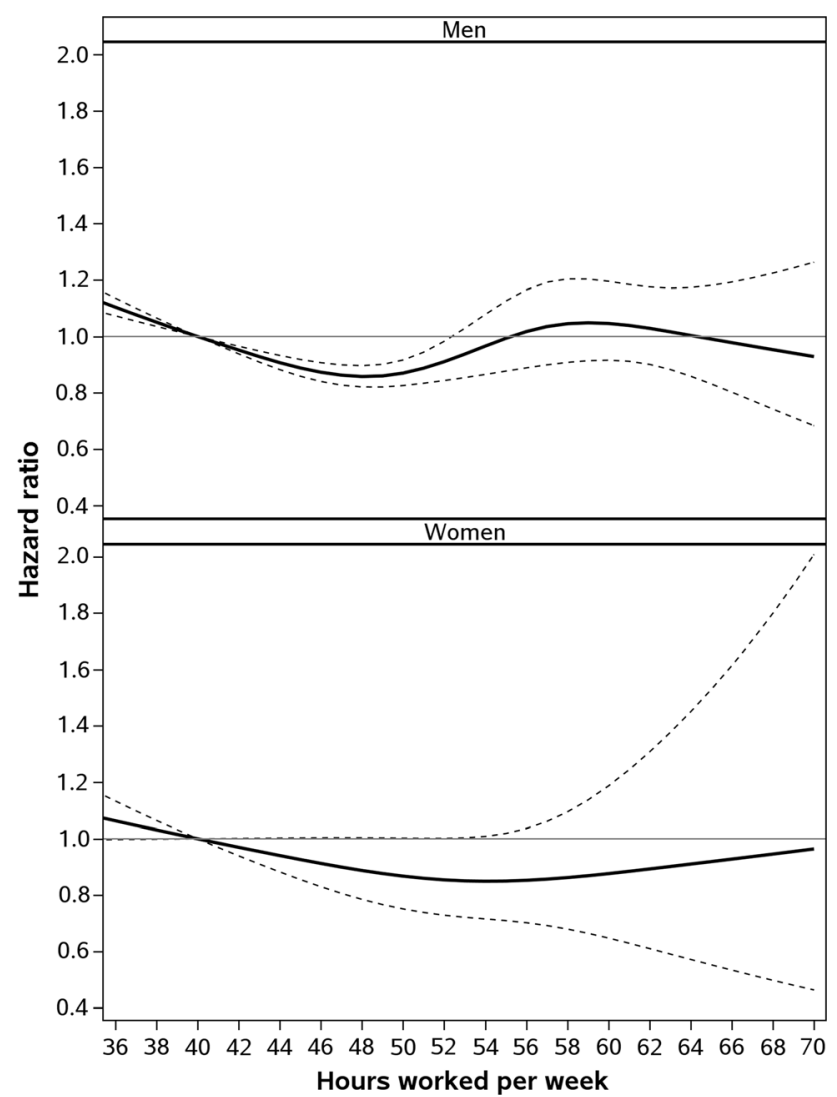

Fig. 1 Exposure-response curves of hours worked per week and cardiovascular disease mortality estimated by multivariable Cox regression models using natural splines with knots fixed at equally spaced intervals of exposure (four knots for men and three for women) and including terms for age, marital status, geographic area of residence, number of dependent children and type of occupation. Dashed lines indicate the 95\% confidence bands. Italy, 2012-2016

Our results are consistent with those found in a cohort study based on 357,085 participants from the Danish Labour Force Survey, where no excess risk of stroke was found with risk ratio for those working $55 \mathrm{~h}$ or over per week of 0.89 (Hannerz et al. 2018a). The same research group did not find an increased risk of ischaemic heart diseases in both men and women working more than $48 \mathrm{~h}$ per week (Hannerz et al. 2018b).

In contrast, other cohort studies reported excess risks. A study based on 6014 office staff, aged 39-61, from 20 London-based Civil Service departments (the Whitehall II cohort) followed up on average for 11 years, reported a $60 \%$ increased risk of incident coronary diseases for 3-4 h of overtime work per day compared with no overtime work. This excess remained even when taking into account socio-economic and lifestyle factors (Virtanen et al. 2010).

A multi-cohort study of 85,494 working men and women from the Individual Participant Data Meta-analysis in Working Populations (IPD-Work) Consortium reported a 1.4-fold increased risk of atrial fibrillation in subjects working more than $55 \mathrm{~h}$ per week as compared with those working standard hours (35-40 h per week) (Kivimaki et al. 2017).

A Danish cohort study, including 5249 men employed in 14 companies in a wide range of industrial sectors with 30-year follow-up, found an excess mortality associated with overtime working ( $\geq 46 \mathrm{~h}$ per week) only in workers with low physical fitness as defined from heart rate during bicycle work, whereas no excess mortality was found among workers with intermediate or high physical fitness (Holtermann et al. 2010). This last finding has been attributed to the higher ability of workers physically trained to recover from work-related stress and to reduce physiological response to work-related stress with lower increase in blood pressure and heart rate.

An excess mortality from all CVD, ischaemic heart diseases and cerebrovascular diseases was also found among men with routine occupations working 55 or more hours per week in a census-based cohort study from Northern Ireland including 414,949 workers who were followed up from 2001 to 2009 (O'Reilly and Rosato 2013). In contrast, no excess risk was found among managers, professionals, intermediate and own account occupations in men, while managers, professionals and own account women showed an excess mortality with HRs of 1.42 and 3.41, respectively, though the limited number of deaths resulted in wide confidence intervals.

The increased mortality in long hours female workers suggested by our results was also found in a previous cohort study on 22,086 women enrolled in the Women's Health Study that found a 38\% excess risk for cardiovascular events in women reporting high job strain or high demand and high control (Slopen et al. 2012). In addition, some studies suggested that women are more vulnerable to work-related stress (Kunz-Ebrecht et al. 2004; Lindeberg et al. 2011; Radi et al. 2005; Rosvall et al. 2002). A study based on 227 men and women recruited in the Whitehall II prospective epidemiological study showed that women have a higher physiological stress response, as measured by salivary cortisol awaking response, during work days than men (Kunz-Ebrecht et al. 2004). In a sample of 5021 Swedish vocationally active individuals, exhaustion in the work context was found more frequently among women than men (Lindeberg et al. 2011). Moreover, a stronger relationship among women than men was found between job constraints and hypertension (Radi et al. 2005) as well as among high job strain (high demands and low decision), atherosclerotic plaque and intimal medial thickness in the carotid bifurcation area (Rosvall et al. 2002).

In our study, CVD mortality risk slightly decreased among individuals working $41-48 \mathrm{~h}$ and $49-54 \mathrm{~h}$ per week as compared to those working $35-40 \mathrm{~h}$ and increased thereafter. This relationship was also observed in the 


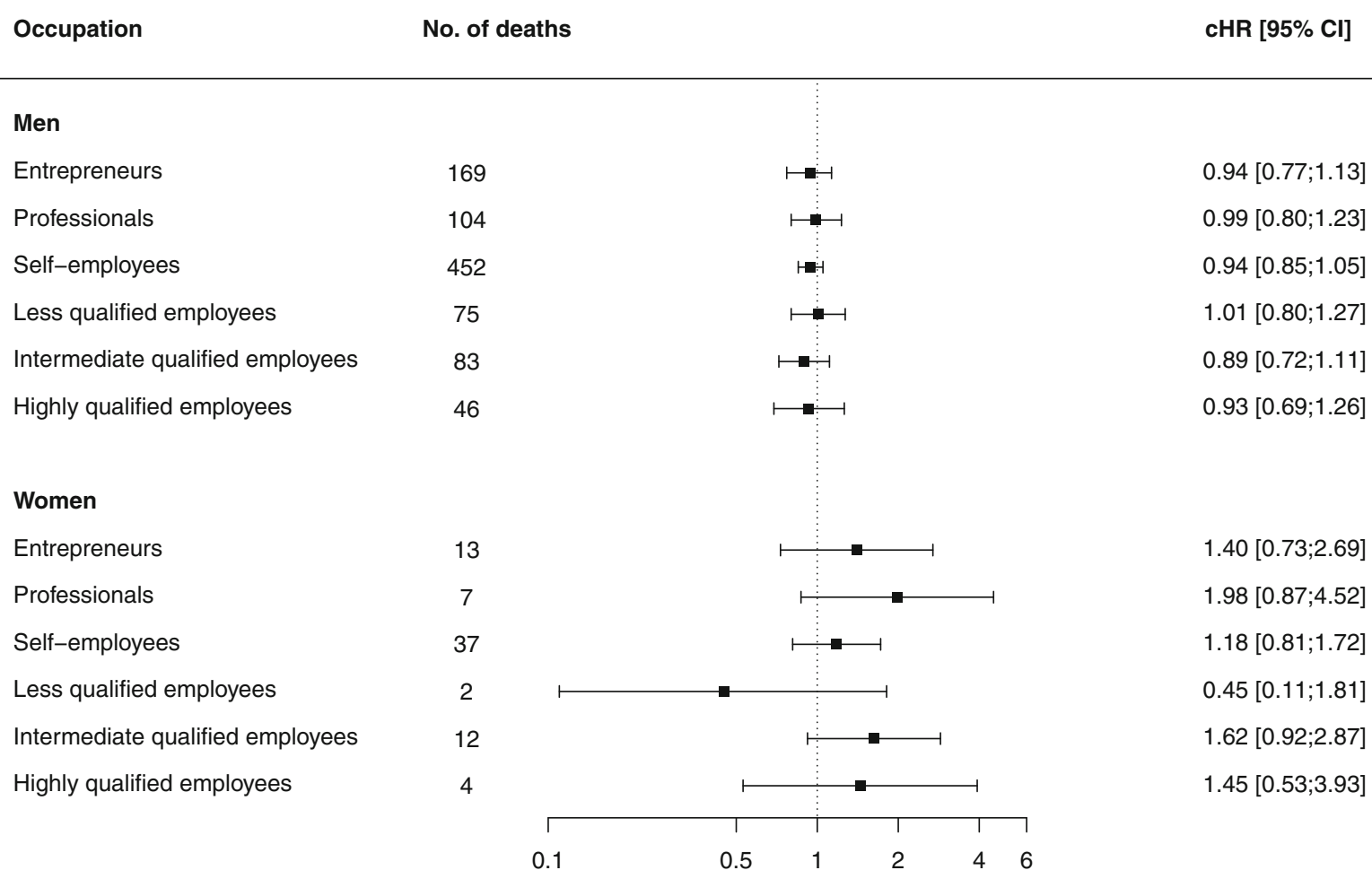

Fig. 2 Number of deaths from cardiovasular diseases in the exposed category (55 or more hour worked per week), cause-specific hazard ratios (cHRs) and 95\% confidence interval (CI) for working 55 or more hours per week as compared to $35-40 \mathrm{~h}$ by type of occupation.
The cHRs were obtained from Cox regression models including terms for category of hours worked per week, age, marital status, geographic area of residence, number of dependent children and type of occupation. Italy, 2012-2016
Danish population for all-cause mortality (Hannerz and Soll-Johanning 2018).

In addition, we did not find an excess risk in women with dependent children. This finding is in contrast with the hypothesis that working women are exposed to higher levels of stress than men due to tension between career, family commitments and caregiving and then to high risk of CVD (Dixon et al. 1991). This may reflect the recent increase in labour market participation of women in Italy and also the cultural changes that led to a more equal sharing, within the couple, of the psychological burden related to combining work and family demands. In contrast to other studies that found a positive association between parity and CVD (Durazo et al. 2018; Ness et al. 1993), in our sample having a child had a protective effect on CVD mortality. Thus, it could be speculated that in a country with very low fertility rates, having a child could even attenuate the CVD risk associated to job strain.

The lack of information on lifestyle risk factors, including smoking, is among the main limitations of the study. This information was not collected in the Italian census archive, but it could be among the mediators of the association between overtime work and CVD mortality among women. In addition, we did not have any follow-up information regarding occupation; thus, we could not evaluate job career and quantify the time during which the subject has been exposed to overtime work.

This large cohort study with more than 11 million workers and around 17000 deaths from CVD provided a huge amount of data allowing to precisely estimate the effect of long working hours on CVD mortality and to evaluate the sex-specific relationship. Most of the studies carried out in this area did not analyse separately men and women, despite the wide sex differences in biological mechanisms, epidemiology of CVDs and prevalence of some psychosocial states that have been identified as coronary risk factors (Low et al. 2010; Schiebinger 2014).

In conclusion, this study does not support the hypothesis of an association between long working hours and increased rates of CVD mortality among active Italian men. It supports, however, the hypothesis that moderately long working hours (41-54 h a week) are associated with a slight decrease in CVD mortality among men. The possible excess risk found among women requires further studies evaluating the role of work-related factors in CVD among women. 
Authors' contribution GA, LF and PB conceived the study. GA and PB performed the data analysis and drafted the manuscript. All authors contributed to the interpretation of the results.

Funding This study did not receive any funding.

\section{Compliance with ethical standards}

Conflicts of interest The authors declare that they have no conflict of interest.

Ethical approval This study is part of an extensive project of the Italian National Institute of Statistics based on the record linkage of pre-existing data. Data were anonymized and irreversibly de-identified after the linkage. The project is included in the National Statistical Program and was approved by the Italian Data Protection Authority.

\section{References}

Alicandro G, Frova L, Sebastiani G, El Sayed I, Boffetta P, La Vecchia C (2017) Educational inequality in cancer mortality: a record linkage study of over 35 million Italians. Cancer Causes Control 28:997-1006. https://doi.org/10.1007/s10552-017-0930$\mathrm{y}$

Chandola T, Brunner E, Marmot M (2006) Chronic stress at work and the metabolic syndrome: prospective study. BMJ 332:521-525. https://doi.org/10.1136/bmj.38693.435301.80

Chandola T, Britton A, Brunner E et al (2008) Work stress and coronary heart disease: what are the mechanisms? Eur Heart J 29:640-648. https://doi.org/10.1093/eurheartj/ehm584

Dixon JP, Dixon JK, Spinner JC (1991) Tensions between career and interpersonal commitments as a risk factor for cardiovascular disease among women. Women Health 17:33-57. https://doi.org/ 10.1300/J013v17n03_03

Doll R, Smith PG, Waterhouse JAH, et al (1982) Comparison between registries: age-standardized rates. Vol. IV. IARC Sci Publ No. 42. In: Cancer incidence in five continents, pp 671-675

Durazo EM, de Baca TC, Slopen N et al (2018) Parity, Job Strain, and Cardiovascular Risk in the Women's Health Study. Curr Cardiovasc Risk 12:8. https://doi.org/10.1007/s12170-018-0571-z

Grosch JW, Caruso CC, Rosa RR, Sauter SL (2006) Long hours of work in the U.S.: associations with demographic and organizational characteristics, psychosocial working conditions, and health. Am J Ind Med 49:943-952. https://doi.org/10.1002/ajim.20388

Hannerz H, Soll-Johanning H (2018) Working hours and all-cause mortality in relation to the EU Working Time Directive: a Danish cohort study. Eur J Public Health 28:810-814. https:// doi.org/10.1093/eurpub/cky027

Hannerz H, Albertsen K, Burr H et al (2018a) Long working hours and stroke among employees in the general workforce of Denmark. Scand J Public Health 46:368-374. https://doi.org/10. $1177 / 1403494817748264$

Hannerz H, Larsen AD, Garde AH (2018b) Long weekly working hours and ischaemic heart disease: a follow-up study among 145 861 randomly selected workers in Denmark. BMJ Open 8:e019807. https://doi.org/10.1136/bmjopen-2017-019807

Holtermann A, Mortensen OS, Burr H, Sogaard K, Gyntelberg F, Suadicani P (2010) Long work hours and physical fitness: 30 -year risk of ischaemic heart disease and all-cause mortality among middle-aged Caucasian men. Heart 96:1638-1644. https://doi.org/10.1136/hrt.2010.197145

Kivimaki M, Kawachi I (2015) Work Stress as a Risk Factor for Cardiovascular Disease. Curr Cardiol Rep 17:630. https://doi. org/10.1007/s11886-015-0630-8

Kivimaki M, Jokela M, Nyberg ST et al (2015a) Long working hours and risk of coronary heart disease and stroke: a systematic review and meta-analysis of published and unpublished data for 603,838 individuals. Lancet 386:1739-1746. https://doi.org/10. 1016/S0140-6736(15)60295-1

Kivimaki M, Singh-Manoux A, Nyberg S, Jokela M, Virtanen M (2015b) Job strain and risk of obesity: systematic review and meta-analysis of cohort studies. Int $\mathrm{J}$ Obes (Lond) 39:1597-1600. https://doi.org/10.1038/ijo.2015.103

Kivimaki M, Nyberg ST, Batty GD et al (2017) Long working hours as a risk factor for atrial fibrillation: a multi-cohort study. Eur Heart J 38:2621-2628. https://doi.org/10.1093/eurheartj/ehx324

Kunz-Ebrecht SR, Kirschbaum C, Marmot M, Steptoe A (2004) Differences in cortisol awakening response on work days and weekends in women and men from the Whitehall II cohort. Psychoneuroendocrinology 29:516-528

Lindeberg SI, Rosvall M, Choi B et al (2011) Psychosocial working conditions and exhaustion in a working population sample of Swedish middle-aged men and women. Eur J Public Health 21:190-196. https://doi.org/10.1093/eurpub/ckq039

Low CA, Thurston RC, Matthews KA (2010) Psychosocial factors in the development of heart disease in women: current research and future directions. Psychosom Med 72:842-854. https://doi.org/ 10.1097/PSY.0b013e3181f6934f

Ness RB, Harris T, Cobb J et al (1993) Number of pregnancies and the subsequent risk of cardiovascular disease. $\mathrm{N}$ Engl $\mathrm{J}$ Med 328:1528-1533. https://doi.org/10.1056/NEJM199305273282104

Nyberg ST, Fransson EI, Heikkila K et al (2013) Job strain and cardiovascular disease risk factors: meta-analysis of individualparticipant data from 47,000 men and women. PLoS ONE 8:e67323. https://doi.org/10.1371/journal.pone.0067323

O'Reilly D, Rosato M (2013) Worked to death? A census-based longitudinal study of the relationship between the numbers of hours spent working and mortality risk. Int $\mathrm{J}$ Epidemiol 42:1820-1830. https://doi.org/10.1093/ije/dyt211

Radi S, Lang T, Lauwers-Cances V et al (2005) Job constraints and arterial hypertension: different effects in men and women: the IHPAF II case control study. Occup Environ Med 62:711-717. https://doi.org/10.1136/oem.2004.012955

Rosvall M, Ostergren PO, Hedblad B, Isacsson SO, Janzon L, Berglund G (2002) Work-related psychosocial factors and carotid atherosclerosis. Int J Epidemiol 31:1169-1178. https:// doi.org/10.1093/ije/31.6.1169

Schiebinger L (2014) Scientific research must take gender into account. Nature 507:9. https://doi.org/10.1038/507009a

Slopen N, Glynn RJ, Buring JE, Lewis TT, Williams DR, Albert MA (2012) Job strain, job insecurity, and incident cardiovascular disease in the Women's Health Study: results from a 10-year prospective study. PLoS ONE 7:e40512. https://doi.org/10.1371/ journal.pone.0040512

Steptoe A, Kivimaki M (2013) Stress and cardiovascular disease: an update on current knowledge. Annu Rev Public Health 34:337-354. https://doi.org/10.1146/annurev-publhealth-031912-114452

Virtanen M, Ferrie JE, Singh-Manoux A et al (2010) Overtime work and incident coronary heart disease: the Whitehall II prospective cohort study. Eur Heart J 31:1737-1744. https://doi.org/10.1093/ eurheartj/ehq124

Publisher's Note Springer Nature remains neutral with regard to jurisdictional claims in published maps and institutional affiliations. 